\title{
The expression of vasohibin- 1 and its prognostic significance in bladder cancer
}

\author{
BO ZHANG ${ }^{1-3^{*}}$, ZHOULIANG WU ${ }^{1,3^{*}}$, WANQIN XIE ${ }^{4}$, DAWEI TIAN ${ }^{1,3}$, \\ FEIRAN CHEN ${ }^{1,3}$, CHUAN QIN ${ }^{1,3}$, ZHIYONG DU ${ }^{1,3}$, GANG TANG $^{1,3}$, QIONGQIONG GAO ${ }^{5}$, \\ XIAOYU QIU ${ }^{6}$, CHANGLI WU ${ }^{1,3}$, JING TIAN ${ }^{2,3}$ and HAILONG HU ${ }^{1,3}$ \\ Departments of ${ }^{1}$ Urology and ${ }^{2}$ Ultrasound, Second Hospital of Tianjin Medical University, Tianjin 300211; \\ ${ }^{3}$ Tianjin Key Laboratory of Urology, Tianjin Institute of Urology; ${ }^{4}$ Key Laboratory of Genetics and Birth Health of Hunan, \\ The Family Planning Research Institute of Hunan, Changsha, Hunan 410126; \\ ${ }^{5}$ Department of Thoracic Oncology, Tianjin Medical University Cancer Institute and Hospital; \\ ${ }^{6}$ College of Management and Economics, Tianjin University, Tianjin 300211, P.R. China
}

Received September 29, 2016; Accepted May 19, 2017

DOI: $10.3892 /$ etm.2017.4969

\begin{abstract}
Angiogenesis is important in the development of solid tumors. Vasohibin-1 (VASH-1) is an endothelium-derived protein that acts as an inhibitor of angiogenesis in many different types of cancer. However, the expression of VASH-1 and its clinical value in bladder cancer remain unknown. The current study analyzed the expression of VASH-1, as well as the expression of the angiogenesis-related factors vascular endothelial growth factor-A, hypoxia inducible factor- $1 \alpha$ and cluster of differentiation 34 in bladder cancer tissues from 50 patients using immunohistochemistry. The associations between the expression of these factors and the clinicopathological characteristics of the patients were assessed. The current study demonstrated that VASH-1 is primarily expressed in the cytoplasm of bladder cancer cells and in a fraction of vascular endothelial cells. Furthermore, the expression of VASH-1 was positively associated with the tumor stage $(\mathrm{P}<0.01)$, pathological grade $(\mathrm{P}<0.01)$ and distant metastasis $(\mathrm{P}<0.05)$ but not with patient age or sex $(\mathrm{P}>0.05)$. Spearman rank correlation tests indicated that levels of those four factors were positively correlated with each other. Kaplan-Meier
\end{abstract}

Correspondence to: Dr Jing Tian, Department of Ultrasound, Second Hospital of Tianjin Medical University, 23 Pingjiang Road, Tianjin 300211, P.R. China

E-mail: 13821777622@163.com

Dr Hailong Hu, Department of Urology, Second Hospital of Tianjin Medical University, 23 Pingjiang Road, Tianjin 300211, P.R. China

E-mail: hhllove2004@163.com

*Contributed equally

Key words: angiogenesis, immunohistochemistry, prognosis, urinary bladder neoplasms, vasohibin-1 analysis indicated that high expression of these four factors was significantly associated with lower 5-year overall survival and progression-free survival rates. Collectively, the results of the current study suggest that VASH-1 is clinically significant in bladder cancer and its high expression may predict the progression and prognosis of patients with bladder cancer. The present study also implies that VASH-1 may be a novel target for vascular targeting therapy.

\section{Introduction}

Bladder cancer is the most common malignant tumor of the urinary system (1). In 2015, bladder cancer was the fourth most common malignant neoplasm in the United States and was one of the leading causes of cancer-associated mortality (2). In developing countries such as China, the incidence of bladder cancer has been steadily increasing, becoming a greater public health problem in these countries $(3,4)$. There are three pathological types of bladder cancer: Urothelial carcinoma, squamous cell carcinoma and adenocarcinoma, with urothelial carcinoma accounting for $>90 \%$ of all cases of bladder cancer (5). Characteristically, 50-70\% of patients initially diagnosed with non-muscle invasive bladder cancer will experience recurrence within 5 years and $\sim 10 \%$ will progress to invasive bladder cancer (6). Thus, it is important to identify biomarkers able to predict disease progression for patients with bladder cancer.

It is well established that angiogenesis is required for the development of solid tumors in different types of cancer. When solid tumor cell clumps reach 1-2 $\mathrm{mm}$ in diameter, angiogenesis results in the formation of new blood vessels that provide adequate oxygen and nutrients for the tumor (7). These newly formed vessels not only promote tumor growth but also facilitate the entry of tumor cells into the circulation, as well as tumor metastasis to distant organs. During angiogenesis, cancer cells produce increased levels of angiogenic factors, overwhelming the effects of angiogenesis inhibitors, to support the growth of new vessels (8). The angiogenesis 
stimulators vascular endothelial growth factor (VEGF) and hypoxia inducible factor- $1 \alpha(\mathrm{HIF}-1 \alpha)$, as well as the inhibitory factors platelet factor 4, endostatin and angiostatin, are being extensively investigated to determine their effects on cancer prognosis and therapy $(9,10)$.

Vasohibin-1 (VASH-1) is as an endothelium-derived protein that is induced by VEGF and fibroblast growth factor-2 (FGF-2) (11). VASH-1 is characterized as a negative feedback regulator of angiogenesis, which may inhibit the migration and proliferation of endothelial cells, though this inhibitory effect is not identical in different endothelial cells (12). Previous studies have demonstrated that VASH-1 is associated with a number of malignant tumors, including colorectal cancer (13), hepatocellular carcinoma (14), non-small cell lung cancer (15), renal cell carcinoma $(16,17)$ and prostate cancer $(18)$. However, the expression of VASH-1 in bladder cancer has not yet been investigated.

The present study aimed to determine the expression of different angiogenesis-related factors including VASH-1, VEGF-A, HIF-1 $\alpha$ and cluster of differentiation (CD)34 in urothelial bladder cancer tissues, identify their associations with various clinicopathological features and their influence on the prognosis of patients with bladder cancer.

\section{Patients and methods}

Patients and tissue samples. A total of 50 patients (41 males and 9 females), with a median age of 67 years (range, 43-81 years) histopathologically diagnosed with urothelial bladder cancer at the Department of Urology, Second Hospital of Tianjin Medical University (Tianjin, China) between January 2010 and January 2011 were recruited in the current study. Tissue samples were collected immediately following transurethral resection of the bladder tumor or radical cystectomy surgery. No patients had received radio- or chemotherapy prior to surgery. Tumor stage was classified according to the seventh edition of the tumor node metastasis classification by the Union for International Cancer Control in 2009 (19) and tumor grade was classified according to the criteria of 2004 World Health Organization classification (20). Regarding tumor stage, there were 19 patients with Ta tumors, 14 patients with T1 tumors, 8 patients with T2 tumors, 6 patients with T3 tumors and 3 patients with T4 tumors. There were 35 patients with low pathological grade tumors and 15 patients with high pathological grade tumors. Distant metastasis was detected in 2 patients (liver metastases); there was no evidence of distant metastasis in the other 48 patients. The present study was approved by the Ethics Committee of Second Hospital of Tianjin Medical University and written informed consent was received from all patients.

Immunohistochemistry. Bladder cancer tissues were surgically removed and fixed in $10 \%$ neutral formalin solution immediately at room temperature for $24 \mathrm{~h}$. Tissues were subsequently embedded in paraffin. Tissue sections $4 \mu \mathrm{m}$-thick were prepared following standard protocols. Sections were then subjected to a $60^{\circ} \mathrm{C}$ water-bath in $0.01 \mathrm{M}$ citric buffer ( $\mathrm{pH}$ 6.0) for $15 \mathrm{~min}$ to perform epitope retrieval. A Polink-1 HRP DAB Detection System kit (PV-6000; ZSGB-Bio Technology, Co., Ltd., Beijing, China) was used to process the tissue sections prior to VASH-1, VEGF-A, HIF-1 $\alpha$ and CD34 immunoreaction. Firstly, to quench endogenous peroxidase activity, tissue sections was incubated with $3 \%$ hydrogen peroxide at room temperature for $5 \mathrm{~min}$, follwed by washing with PBS for $3 \mathrm{~min}$. Subsequently, mouse monoclonal anti-VASH-1 antibody (dilution, 1:100; sc-365541; Santa Cruz Biotechnology, Inc., Dallas, TX USA), rabbit polyclonal anti-VEGF-A antibodies (dilution, 1:100; ab51745; Abcam, Cambridge, UK), mouse monoclonal anti-HIF-1 $\alpha$ antibody (dilution, 1:200; ab16066; Abcam) and mouse monoclonal anti-CD34 antibody (dilution, 1:100; ZM-0046; ZSGB-Bio Technology, Co., Ltd.) were used to treat tissue sections overnight at $4^{\circ} \mathrm{C}$ in a humidified chamber. To determine specific antigen-antibody interaction, PBS was used as a negative control in the primary incubation step. The following day, tissue sections were washed with PBS for 3 min and incubated with rabbit/mouse immunoglobulin G/horseradish peroxidase (HRP) polymer at $37^{\circ} \mathrm{C}$ for $15 \mathrm{~min}$. Finally, after 3 min PBS washed, the sections were colored and counterstained using a DAB kit (ZLI-9017; ZSGB-Bio Technology, Co., Ltd.) at room temperature for $2 \mathrm{~min}$ and hematoxylin at room temperature for $30 \mathrm{sec}$.

Evaluation of immunohistochemical staining. The expression of VASH-1, VEGF-A, and HIF-1 $\alpha$ in bladder cancer tissues was evaluated using Image Pro Plus 6.0 (Media Cybernetics, Inc., Rockville, MD, USA). The software was used to measure integrated optical density (IOD) and the area of pictures, which were subsequently used to calculate average optical density (AOD) using the following formula: AOD=IOD/Area. CD34 expression was determined by evaluating microvessel density (MVD) according to the criteria established by Weidner (21). A tissue sample was first examined using a light microscope at low magnification (x100) under a light microscope to identify the highest vascular density area (hot spot). Subsequently, five areas of the highest density were selected under a higher magnification (x200) for counting. The average density of the five areas was recorded as the MVD level for this tissue. During this process, any brown-staining endothelial cell or endothelial cell cluster that was clearly separate from adjacent vessels, tumor cells and other connective tissue elements was considered to be a single countable microvessel, even those distinct clusters of stained endothelial cells which may be from the same vessel. However, vessels with a thick muscular wall or with a lumen $>8$ red blood cells in diameter $(\sim 50 \mu \mathrm{m})$ were excluded from the count. Subsequently, in order to carry out Kaplan-Meier analysis, these patients were divided into the low and high expression groups according to the average values of the AOD or MVD individually.

Follow-up. Following patient discharge, information regarding patient disease progression was received by letter, telephone or outpatient visits every 3 months. The period for the 5 -year overall survival (OS) was estimated from the date of initial surgery to the date of mortality from any cause or the final follow-up session. The 5-years progression-free survival (PFS) duration was calculated from the date of initial surgery to the date of disease recurrence, progression or the final follow-up.

Statistical analysis. SPSS 13.0 (SPSS, Inc., Chicago, IL, USA) was used for statistical analysis and the results were 

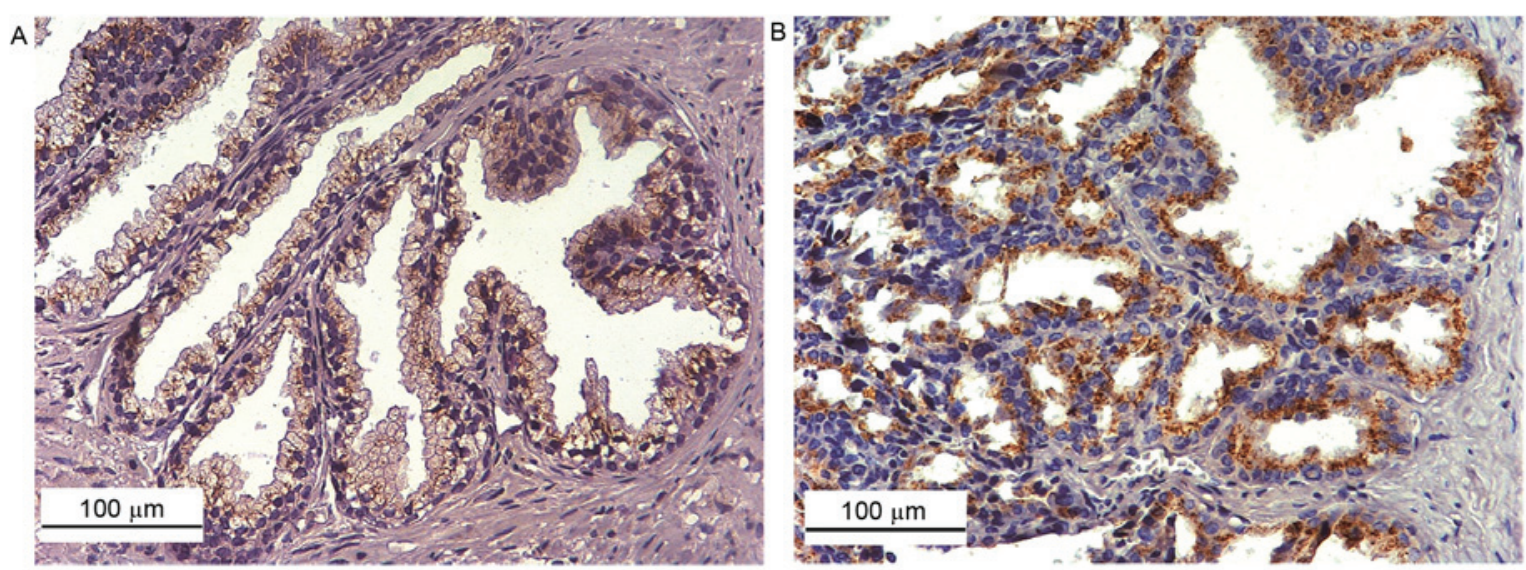

Figure 1. Immunostaining of VASH-1 in bladder cancer (magnification, x200). (A) Low and (B) high expression of VASH-1 in tumor tissues. VASH-1, vasohibin-1.
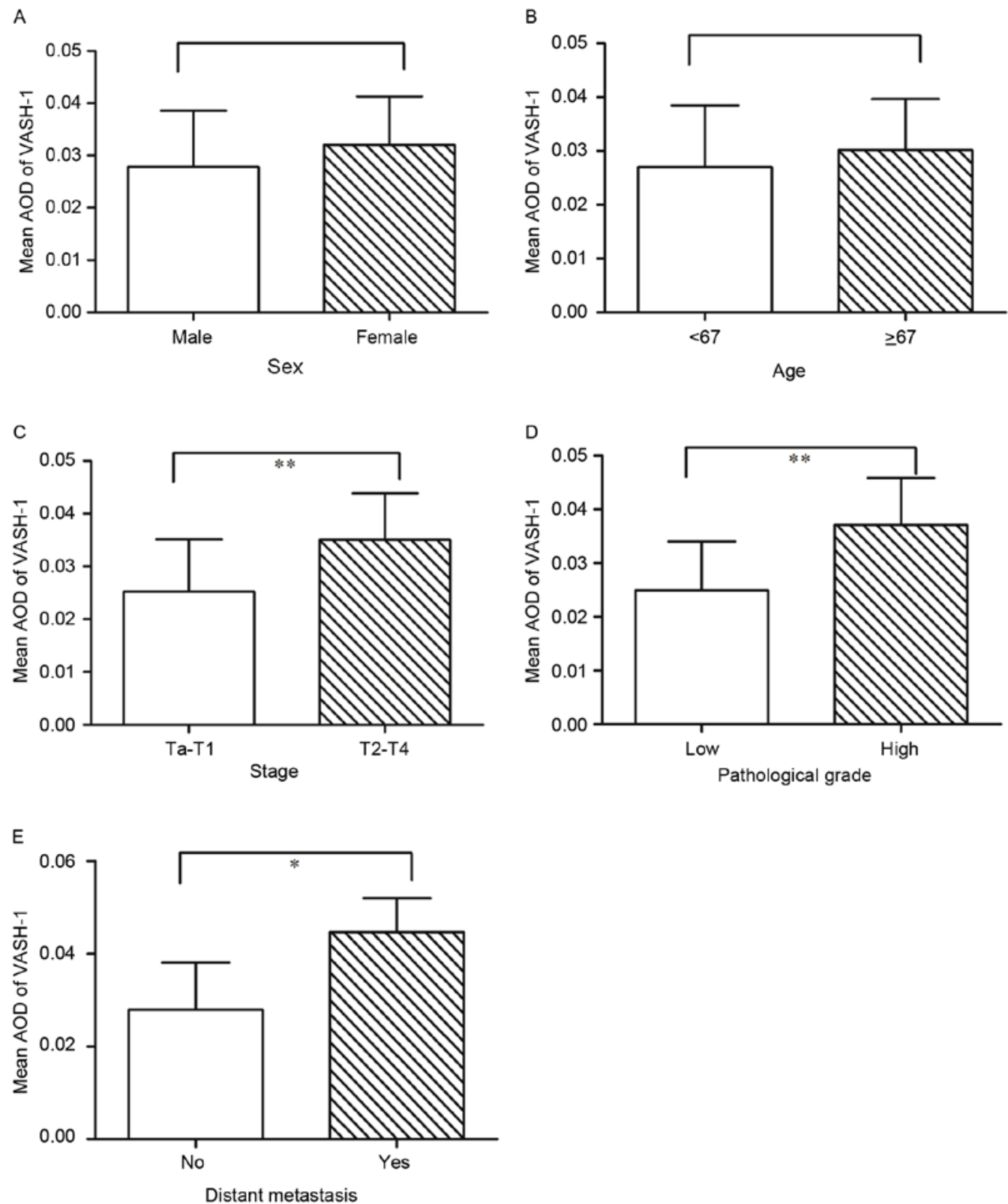

Figure 2. Comparison of VASH-1 expression in bladder cancer tissues. Patients were divided into two groups for analysis of (A) sex, (B) age, (C) tumor stage, (D) pathological grade or (E) distant metastasis. The results were expressed as the mean \pm standard deviation. ${ }^{*} \mathrm{P}<0.05$; ${ }^{* *} \mathrm{P}<0.01$. AOD, average optical density; VASH-1, vasohibin-1. 
Table I. Associations between clinicopathological parameters and VASH-1, VEGF-A, HIF-1 $\alpha$ or CD34 expression in bladder cancer tissues.

\begin{tabular}{|c|c|c|c|c|c|c|c|c|c|}
\hline Parameter & $\begin{array}{l}\text { No. of } \\
\text { cases }\end{array}$ & $\begin{array}{l}\text { VASH-1 } \\
\text { expression }\end{array}$ & P-value & $\begin{array}{l}\text { VEGF-A } \\
\text { expression }\end{array}$ & P-value & $\begin{array}{c}\text { HIF-1 } \alpha \\
\text { expression }\end{array}$ & P-value & $\begin{array}{c}\text { CD34 } \\
\text { expression }\end{array}$ & P-value \\
\hline Sex & & & 0.277 & & 0.36 & & 0.088 & & 0.744 \\
\hline Male & 41 & $0.028 \pm 0.011$ & & $0.130 \pm 0.051$ & & $0.0060 \pm 0.0032$ & & $45.56 \pm 17.74$ & \\
\hline Female & 9 & $0.032 \pm 0.009$ & & $0.148 \pm 0.051$ & & $0.0082 \pm 0.0039$ & & $47.67 \pm 15.84$ & \\
\hline Age & & & 0.282 & & 0.322 & & 0.819 & & 0.18 \\
\hline$<67$ & 25 & $0.027 \pm 0.011$ & & $0.126 \pm 0.055$ & & $0.0063 \pm 0.0036$ & & $42.64 \pm 17.79$ & \\
\hline$\geq 67$ & 25 & $0.030 \pm 0.009$ & & $0.141 \pm 0.046$ & & $0.0065 \pm 0.0033$ & & $49.24 \pm 16.45$ & \\
\hline T stage & & & $<0.01$ & & $<0.01$ & & 0.033 & & $<0.01$ \\
\hline $\mathrm{Ta}-\mathrm{T} 1$ & 33 & $0.025 \pm 0.010$ & & $0.114 \pm 0.049$ & & $0.0056 \pm 0.0034$ & & $41.21 \pm 16.06$ & \\
\hline $\mathrm{T} 2-\mathrm{T} 4$ & 17 & $0.035 \pm 0.009$ & & $0.170 \pm 0.032$ & & $0.0078 \pm 0.0032$ & & $55.12 \pm 16.23$ & \\
\hline Pathological grade & & & $<0.01$ & & $<0.01$ & & $<0.01$ & & $<0.01$ \\
\hline Low & 35 & $0.025 \pm 0.009$ & & $0.119 \pm 0.050$ & & $0.0054 \pm 0.0028$ & & $40.89 \pm 16.00$ & \\
\hline High & 15 & $0.037 \pm 0.009$ & & $0.166 \pm 0.038$ & & $0.0088 \pm 0.0037$ & & $57.73 \pm 14.51$ & \\
\hline Distant metastasis & & & 0.027 & & $<0.01$ & & 0.027 & & 0.086 \\
\hline No & 48 & $0.028 \pm 0.010$ & & $0.130 \pm 0.049$ & & $0.0062 \pm 0.0033$ & & $45.08 \pm 17.02$ & \\
\hline Yes & 2 & $0.045 \pm 0.007$ & & $0.217 \pm 0.003$ & & $0.0116 \pm 0.0016$ & & $66.50 \pm 12.02$ & \\
\hline
\end{tabular}

All results are presented as the mean \pm standard deviation. VASH-1, vasohibin-1; VEGF-A, vascular endothelial growth factor-A; HIF-1 $\alpha$, hypoxia inducible factor- $1 \alpha$; CD34, cluster of differentiation 34 .

expressed as the mean \pm standard deviation. An independent-sample $\mathrm{t}$ test was performed to compare the differences between groups and the correlation between two continuous variables was evaluated by using the Spearman rank correlation test. The 5-year OS and PFS were analyzed using the Kaplan-Meier method and the log-rank test was used to analyze differences between groups. All statistical tests were bilateral and $\mathrm{P}<0.05$ was considered to indicate a statistically significant difference.

\section{Results}

VASH-1 expression in bladder cancer. Immunohistochemistry analysis demonstrated that VASH-1 was mainly expressed in the cytoplasm of cancer cells and in a fraction of vascular endothelial cells (Fig. 1). The mean AOD of VASH-1 was $0.029 \pm 0.011$ in all 50 bladder cancer tissues. The expression of VASH-1 did not differ significantly between males and females (Fig. 2A) and between older and younger patients (Fig. 2B). According to whether there was muscle invasion or not, the 50 patients were classified into two groups: A Ta-T1 group $(n=33)$ and a T2-T4 group $(n=17)$. Notably, the AOD of VASH-1 in the Ta-T1 group was $0.025 \pm 0.010$ and in the T2-T4 group was $0.035 \pm 0.009$; a significant difference $(\mathrm{P}<0.01$; Fig. 2C). Additionally, there was a significant difference in the expression of VASH-1 in terms of pathological grade $(\mathrm{P}<0.01$; Fig. 2D) and distant metastasis $(\mathrm{P}<0.05$; Fig. 2E). Detailed data are presented in Table I.

VEGF-A and HIF-1 $\alpha$ expression in bladder cancer. VEGF-A staining was observed primarily in the cytoplasm and on the membrane of cancer cells, as well as in a proportion of the nucleus of cancer cells and vascular endothelial cells (Fig. 3A and B). HIF-1 $\alpha$ was predominantly expressed in the nucleus of cancer cells (Fig. 3C and D). The average AODs of VEGF-A and HIF- $1 \alpha$ were $0.133 \pm 0.051$ and $0.0064 \pm 0.0034$, respectively, for the cohort in the current study. When patients were dichotomized in terms of clinical parameters ( $\mathrm{T}$ stage, pathological grade or distant metastasis), significant differences were observed between the two groups $(\mathrm{P}<0.05$; Table I). However, there were no significant differences in the mean levels of VEGF-A and HIF-1 $\alpha$ between the two patient groups by either sex or age $(\mathrm{P}>0.05$; Table I).

CD34 is a representative marker for the formation of microvessels. CD34 was primarily expressed in the cytoplasm and on the membranes of vascular endothelial cells (Fig. 3E and F). Mean CD34 was 45.94 17.28 for the cohort. There were significant differences in mean CD34 levels between the two patient groups based on $\mathrm{T}$ stage $(\mathrm{P}<0.01)$ and pathological grade $(\mathrm{P}<0.01)$ but not in distant metastasis, sex or age $(\mathrm{P}>0.05$; Table I).

Correlations between VASH-1, VEGF-A, HIF-1 $\alpha$ and CD34 in bladder cancer. Spearman rank correlation analysis revealed that there were positive correlations between the levels of VASH-1 and VEGF-A ( $\mathrm{r}=0.750)$; VASH-1 and HIF-1 $\alpha$ ( $r=0.626$ ); and VASH-1 and CD34 ( $r=0.637$; all $\mathrm{P}<0.01$; Fig. 4). In addition, the correlation coefficients between VEGF-A and HIF-1 $\alpha$, VEGF-A and CD34, HIF-1 $\alpha$ and CD34 were 0.524, 0.530 , and $0.478(\mathrm{P}<0.01)$, respectively (data not shown).

Prognostic significance of VASH-1,VEGF-A, HIF-1 $\alpha$ and $C D 34$ expression in bladder cancer. All patients were followed 

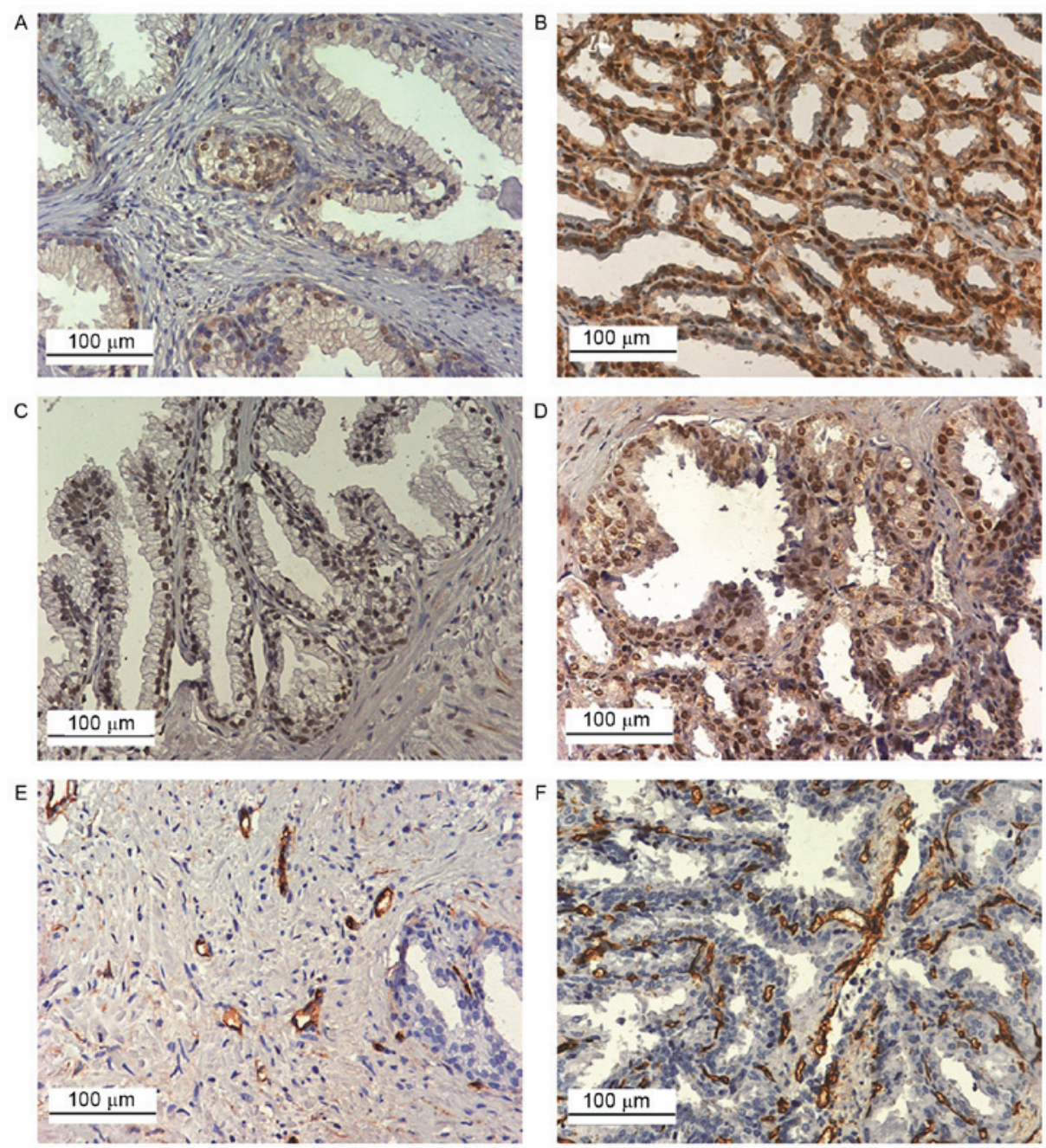

Figure 3. Immunostaining of VEGF-A, HIF-1 $\alpha$ and CD34 in bladder cancer tissues (magnification, x200). Representative images for low expression of (A) VEGF-A, (C) HIF-1 $\alpha$ and (E) CD34 in tumor tissues; representative images for high expression of (B) VEGF-A, (D) HIF-1 $\alpha$ and (F) CD34 in tumor tissues. VEGF-A, vascular endothelial growth factor A; HIF-1 $\alpha$, hypoxia inducible factor-1 $\alpha$; CD34, cluster of differentiation 34.
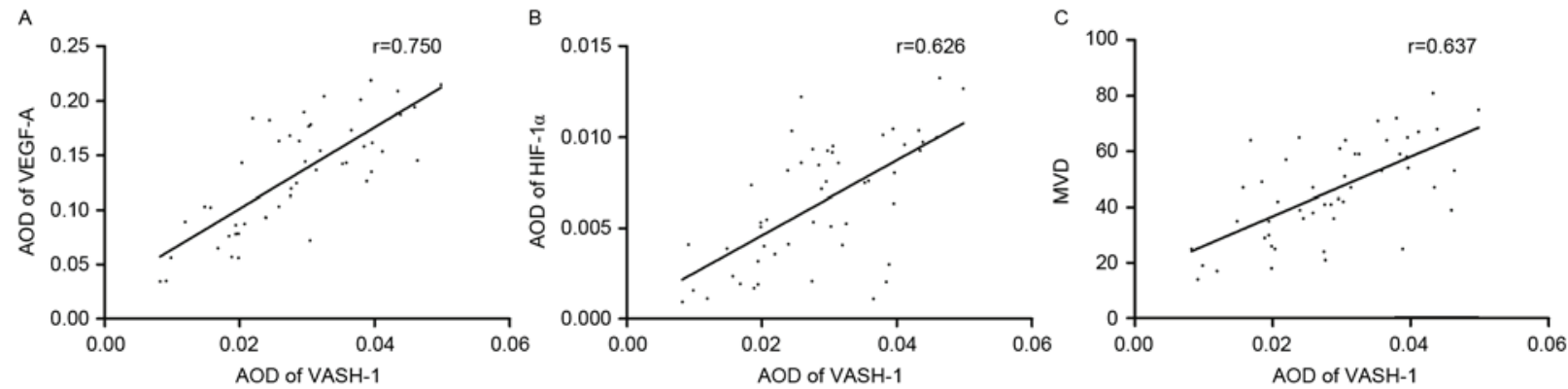

Figure 4. Correlation between the expression of VASH-1, VEGF-A, HIF-1 $\alpha$ and MVD. There were positive correlations between (A) VASH-1 and VEGF-A, (B) VASH-1 and HIF-1 $\alpha$ and (C) VASH-1 and MVD. P<0.01. CD34 expression was used to calculate MVD. VASH-1, vasohibin-1; VEGF-A, vascular endothelial growth factor A; HIF-1 $\alpha$, hypoxia inducible factor-1 $\alpha$; CD34, cluster of differentiation 34; AOD, average optical density; MVD, microvessel density.

up for 5 years. The Kaplan-Meier analysis demonstrated that patients exhibiting high expression of VASH-1, VEGF-A, HIF-1 $\alpha$ and CD34 had significantly lower 5-year OS and PFS rates than patients exhibiting low expression of these factors $(\mathrm{P}<0.05$; Fig. 5). The difference in OS and PFS between patients with high and low expression of VASH-1 was greater $(\mathrm{P}<0.01)$ than the difference in OS and PFS between patients with high and low expression of VEGF-A, HIF-1 $\alpha$ and CD34
$(\mathrm{P}<0.05)$, indicating that the effect of VASH-1 expression on the prognosis of bladder cancer may be greater than that of VEGF-A, HIF-1 $\alpha$ and CD34 expression.

\section{Discussion}

Angiogenesis is critically important for the progression of solid tumors. To date, a number of vasoactive factors have 


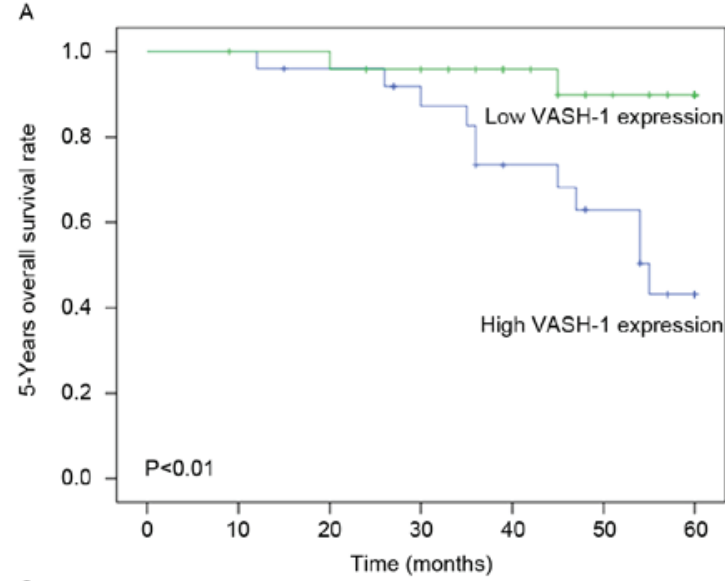

$c$

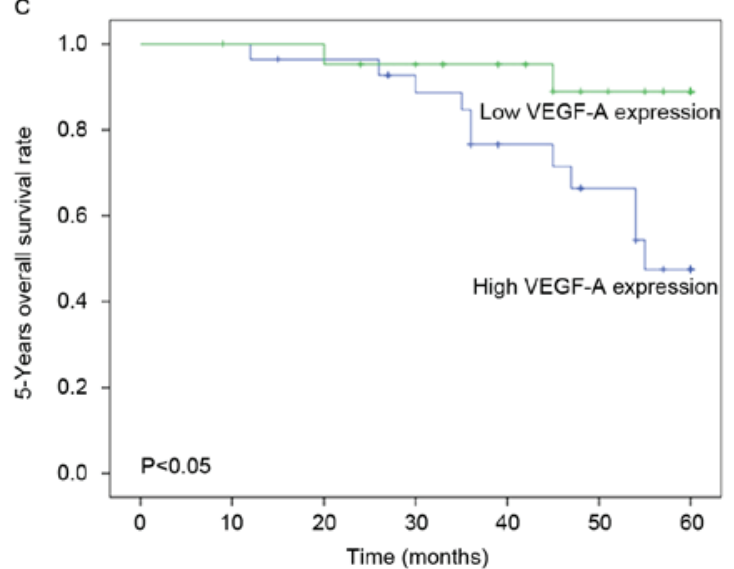

E

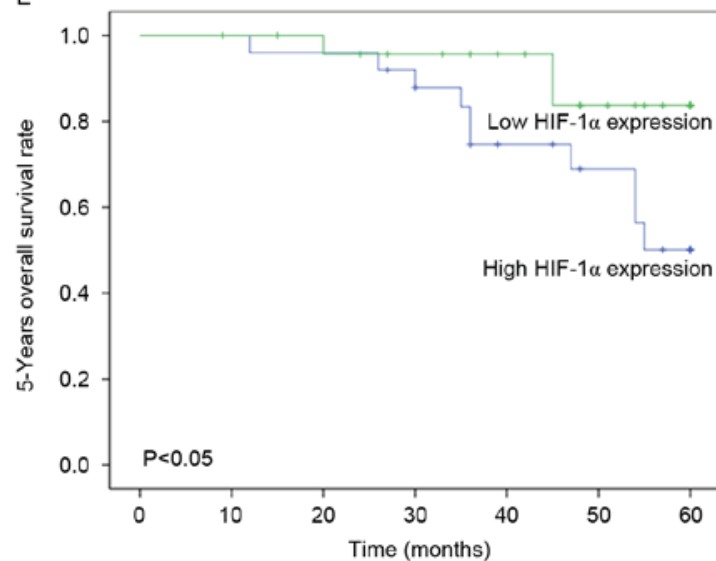

G

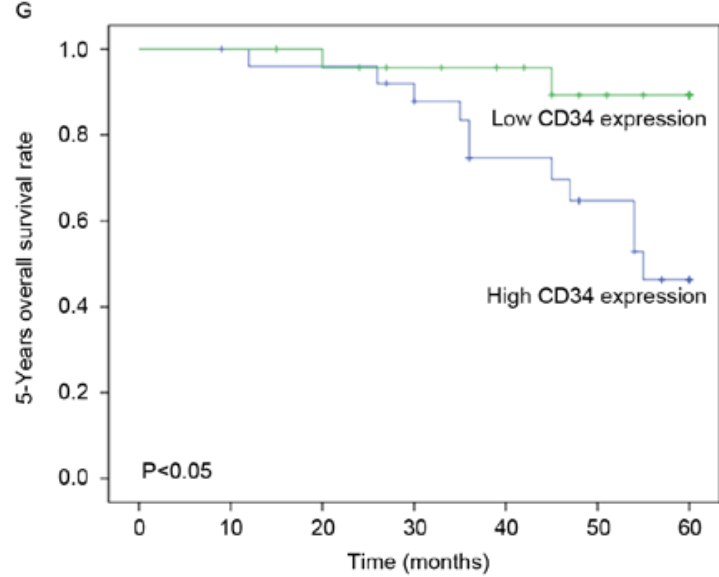

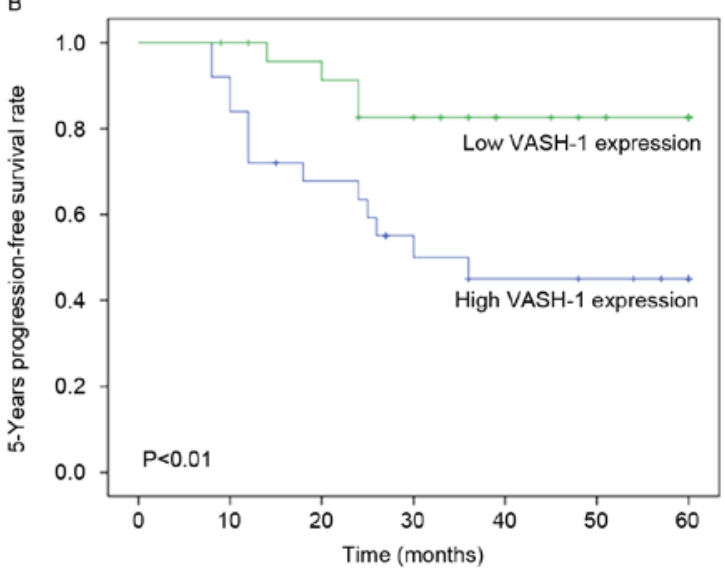
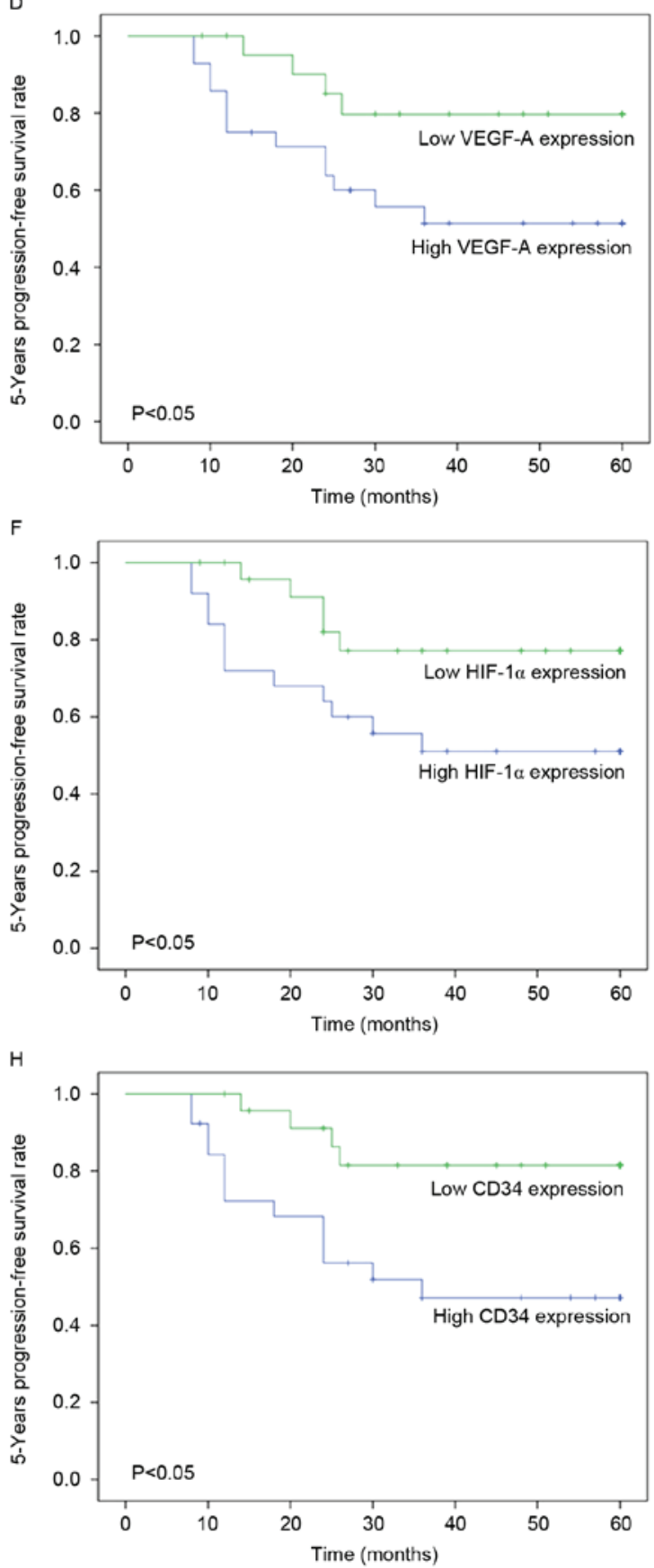

Figure 5. Kaplan-Meier survival curves for 5-year OS and PFS of VASH-1, VEGF-A, HIF-1 $\alpha$ and CD34 in bladder cancer. The 5-year OS and PFS for high expression group of (A and B) VASH-1, (C and D) VEGF-A, (E and F) HIF-1 $\alpha$, and (G and H) CD34 were significantly lower than the low expression group, respectively. VASH-1, vasohibin-1; VEGF-A, vascular endothelial growth factor A; HIF-1 $\alpha$, hypoxia inducible factor-1 $\alpha$; CD34, cluster of differentiation 34; OS, overall survival; PFS, progression-free survival. 
been identified, including VEGF (22). VEGF-A is a representative member of the VEGF family and is able to upregulate vascular endothelial-cadherin, matrix metalloproteases and ephrin type-A receptor 2, which are crucial for the formation of vasculogenic mimicry and the alteration of vascular endothelium to promote tumor growth and invasion, particularly in avascular regions (23). VEGF receptors have 5 different isoforms and VEGF-A primarily recognizes VEGFR1 and VEGFR2 (24). The binding of VEGF to the different receptors may have different biological impacts on angiogenesis. During the development of solid tumors, following tumor enlargement, ischemia, hypoxia and necrosis will occur in the center of the neoplasm (25). In response to growth stresses, HIF-1 $\alpha$ stimulates VEGF expression in tumor cells by binding to the enhancer of the VEGF gene. Consequently, VEGF will promote the formation of new blood vessels (26). To quantify the newly formed blood vessels, it has been suggested that CD34 should be used as a molecular marker to evaluate the MVD within tumors (27).

The VASH-1 gene is located in human chromosome $14 \mathrm{q} 24.3$ and has 8 exons and 7 introns (28). It is able to inhibit angiogenesis under physiological and pathological conditions (29). It has also been demonstrated that VASH-1 serves an important role in many malignant tumors, including renal cell carcinoma $(16,17)$, prostate (18), breast (30) and ovarian cancer (31). However, the behavior and influence of VASH-1 differs in different types of cancer and even among different pathological types of the same cancer. Zhao et al (16) demonstrated that VASH-1 was primarily expressed in the cytoplasm and on the membrane of tumor cells and vascular endothelial cells in renal cell carcinoma and that the expression of VASH-1 in tumor tissues was significantly reduced compared with adjacent non-tumor renal tissues $(\mathrm{P}<0.05)$. However, in prostate cancer, it was demonstrated that VASH-1 was primarily expressed in vascular endothelial cells and its expression was positively correlated with the malignancy degree of the tumor (18). It remains unclear what the reasons for the different behavior of VASH-1 in different types of cancer are. It has been suggested this phenomenon is associated with the local vessel distribution of tumor tissues (tumor cells-vessels or tumor cells-stromal-vessels) and the different regulatory mechanisms of VASH-1 in different tumors.

The current study determined that levels of VASH-1, VEGF-A and HIF-1 $\alpha$ in bladder cancer tissue were significantly associated with a more severe tumor stage and pathological grade, as well as with the presence of distant metastasis $(\mathrm{P}<0.05)$. Notably, CD34 levels were significantly associated with a more severe tumor stage and pathological grade $(\mathrm{P}<0.01)$ but not with the presence of distant metastasis. These results are similar to those of Canoglu et al (32) and Deniz et al (33), which investigated the effect of MVD on bladder cancer. However, neither study investigated the association between MVD and distant metastasis in bladder carcinoma.

The current study used the Spearman correlation test to determine the potential correlations between the vasoactive factors. The correlation coefficients between VASH-1 and VEGF-A, VASH-1 and HIF-1 $\alpha$, VASH-1 and CD34 were $0.750,0.626$ and 0.637 , respectively $(\mathrm{P}<0.01)$, suggesting that the angiogenesis inhibitor VASH-1 may be the most responsive to the alteration of angiogenesis-promoting factors in bladder cancer. As previously mentioned, VASH-1 is induced by VEGF and FGF-2, and HIF-1 $\alpha$ is able to increase the expression of VEGF by promoting the transcription of target genes. Thus, when VEGF or other factors promote tumor angiogenesis, they also promote the production of VASH-1, which acts as an angiogenesis inhibitor and exhibits an anti-tumor effect on the body itself (34). The present study identified that there was a positive correlation between VASH-1 expression and the number of microvessels formed during tumor progression. Therefore, it is hypothesized that although VASH-1 expression inhibits angiogenesis via a negative feedback mechanism, it alone may not be potent enough to dominate the stimulatory factors and there may be other unknown pathways suppressing the activity of VASH-1. Kaplan-Meier analysis demonstrated that the group exhibiting high expression of VASH-1, VEGF-A, HIF-1 $\alpha$ and CD34 had significantly lower 5-year OS and PFS rates than the low expression group. Furthermore, the results of the current study indicated that the effect of VASH-1 on the prognosis of patients with bladder cancer was greater than that of VEGF-A, HIF-1 $\alpha$ and CD34. This suggests that VASH-1 may be more effective at predicting the progression and metastasis of bladder cancer. One major limitation of the current study was that most of the patients were from the north of China, meaning that the results may have regional limitations.

Preventing tumor recurrence and progression in patients with non-muscle invasive bladder cancer following transurethral resection of the bladder tumor remains challenging. In light of the critical role of angiogenesis in tumor development, angiogenesis inhibitors can act to starve neoplasms, thereby inhibiting the growth and recurrence of tumors. A number of anti-angiogenic drugs, such as sunitinib, are already clinically used, however the side effects they induce limit their use in patients. Therefore, the results of the current study may provide new clues to further understand the mechanisms by which VASH-1 suppresses angiogenesis in bladder cancer and exploit the potential of VASH-1 in vascular targeting therapy.

\section{Acknowledgements}

The present study was supported by the Natural Science Foundation of Tianjin City [15JCYBJC27200].

\section{References}

1. Torre LA, Bray F, Siegel RL, Ferlay J, Lortet-Tieulent J and Jemal A: Global cancer statistics, 2012. CA Cancer J Clin 65: 87-108, 2015.

2. Siegel RL, Miller KD and Jemal A: Cancer statistics, 2015. CA Cancer J Clin 65: 5-29, 2015.

3. Chavan S, Bray F, Lortet-Tieulent J, Goodman M and Jemal A: International variations in bladder cancer incidence and mortality. Eur Urol 66: 59-73, 2014.

4. Chen W, Zheng R, Baade PD, Zhang S, Zeng H, Bray F, Jemal A, Yu XQ and He J: Cancer statistics in China, 2015. CA Cancer J Clin 66: 115-132, 2016.

5. Fleshner NE, Herr HW, Stewart AK, Murphy GP, Mettlin C and Menck HR: The National Cancer Data Base report on bladder carcinoma. The American College of Surgeons Commission on Cancer and the American Cancer Society. Cancer 78: 1505-1513, 1996.

6. Ghoneim MA, Abdel-Latif M, el-Mekresh M, Abol-Enein H, Mosbah A, Ashamallah A and el-Baz MA: Radical cystectomy for carcinoma of the bladder: 2,720 consecutive cases 5 years later. J Urol 180: 121-127, 2008. 
7. Clark PR and Hersh EM: Cationic lipid-mediated gene transfer: Current concepts. Curr Opin Mol Ther 1: 158-176, 1999.

8. Kut C, Mac Gabhann F and Popel AS: Where is VEGF in the body? A meta-analysis of VEGF distribution in cancer. Br J Cancer 97: 978-985, 2007.

9. Kerbel RS: Vasohibin: The feedback on a new inhibitor of angiogenesis. J Clin Invest 114: 884-886, 2004.

10. Sato Y: Update on endogenous inhibitors of angiogenesis. Endothelium 13: 147-155, 2006.

11. Watanabe K, Hasegawa Y, Yamashita H, Shimizu K, Ding Y, Abe M, Ohta H, Imagawa K, Hojo K, Maki H, et al: Vasohibin as an endothelium-derived negative feedback regulator of angiogenesis. J Clin Invest 114: 898-907, 2004.

12. Sato Y and Sonoda H: The vasohibin family: A negative regulatory system of angiogenesis genetically programmed in endothelial cells. Arterioscler Thromb Vasc Biol 27: 37-41, 2007.

13. Liu S, Han B, Zhang Q, Dou J, Wang F, Lin W, Sun Y and Peng G: Vasohibin-1 suppresses colon cancer. Oncotarget 6: 7880-7898, 2015.

14. Murakami K, Kasajima A, Kawagishi N, Sekiguchi S, Fujishima F, Watanabe M, Sato Y, Ohuchi N and Sasano H: The prognostic significance of vasohibin 1-associated angiogenesis in patients with hepatocellular carcinoma. Hum Pathol 45: 589-597, 2014.

15. Zhang T, Yu TT, Zhang DM, Hou XM, Liu XJ, Zhao D and Shan L: Vasohibin-1 expression detected by immunohistochemistry correlates with prognosis in non-small cell lung cancer. Med Oncol 31: 963, 2014

16. Zhao G, Yang Y, Tang Y, Han R and Sun Y: Reduced expression of vasohibin-1 is associated with clinicopathological features in renal cell carcinoma. Med Oncol 29: 3325-3334, 2012.

17. Kanomata N, Sato Y, Miyaji Y, Nagai A and Moriya T: Vasohibin-1 is a new predictor of disease-free survival in operated patients with renal cell carcinoma. J Clin Pathol 66: 613-619, 2013.

18. Kosaka T, Miyazaki Y, Miyajima A, Mikami S, Hayashi Y, Tanaka N, Nagata H, Kikuchi E, Nakagawa K, Okada Y, et al: The prognostic significance of vasohibin-1 expression in patients with prostate cancer. Br J Cancer 108: 2123-2129, 2013.

19. Sobin LH, Cospodariwicz M and Wittekind C (eds): UICC international union against cancer. 7th edition. Wiley-Blackwell, New York, NY, 2009.

20. World Health Organization Classification of Tumours: Pathology and Genetics of Tumours of the Urinary System and Male Genita Organs. Eble JN, Sauter G, Epstein JI and Sesterhenn IA (eds) IARC Press, Lyon, 2004

21. Weidner N: Intratumor microvessel density as a prognostic factor in cancer. Am J Pathol 147: 9-19, 1995.

22. Aragon-Ching JB and Dahut WL: VEGF inhibitors and prostate cancer therapy. Curr Mol Pharmacol 2: 161-168, 2009.
23. Qiao L, Liang N, Zhang J, Xie J, Liu F, Xu D, Yu X and Tian Y: Advanced research on vasculogenic mimicry in cancer. J Cell Mol Med 19: 315-326, 2015.

24. Gram A, Hoffmann B, Boos A and Kowalewski MP: Expression and localization of vascular endothelial growth factor A (VEGFA) and its two receptors (VEGFR1/FLT1 and VEGFR2/FLK1/KDR) in the canine corpus luteum and utero-placental compartments during pregnancy and at normal and induced parturition. Gen Comp Endocrinol 223: 54-65, 2015

25. Harris AL: Hypoxia-A key regulatory factor in tumour growth Nat Rev Cancer 2: 38-47, 2002.

26. Zhang TY, Yang JL and Huo BJ: Effect of overexpression of hypoxia-inducible factor- $1 \alpha$ induced by hyperoxia in vivo in LNCaP tumors on tumor growth rate. Asian Pac J Trop Med 8: 813-820, 2015.

27. Miyata Y, Mitsunari K, Asai A, Takehara K, Mochizuki Y and Sakai H: Pathological significance and prognostic role of microvessel density, evaluated using CD31, CD34, and CD105 in prostate cancer patients after radical prostatectomy with neoadjuvant therapy. Prostate 75: 84-91, 2015.

28. Shibuya T, Watanabe K, Yamashita H, Shimizu K, Miyashita H, Abe M, Moriya T, Ohta H, Sonoda H, Shimosegawa T, et al: Isolation and characterization of vasohibin-2 as a homologue of VEGF-inducible endothelium-derived angiogenesis inhibitor vasohibin. Arterioscler Thromb Vasc Biol 26: 1051-1057, 2006.

29. Kern J, Bauer M, Rychli K, Wojta J, Ritsch A, Gastl G, Gunsilius E and Untergasser G: Alternative splicing of vasohibin-1 generates an inhibitor of endothelial cell proliferation, migration, and capillary tube formation. Arterioscler Thromb Vasc Biol 28 478-484, 2008

30. Tamaki K, Moriya T, Sato Y, Ishida T, Maruo Y, Yoshinaga K, Ohuchi N and Sasano H: Vasohibin-1 in human breast carcinoma: A potential negative feedback regulator of angiogenesis. Cancer Sci 100: 88-94, 2009.

31. Takahashi Y, Saga Y, Koyanagi T, Takei Y, Machida S, Taneichi A, Mizukami H, Sato Y, Matsubara S and Fujiwara H: Vasohibin-1 expression inhibits advancement of ovarian cancer producing various angiogenic factors. Cancer Sci 107: 629-637, 2016.

32. Canoglu A, Göğüuş C, Beduk Y, Orhan D, Tulunay O and Baltaci S: Microvessel density as a prognostic marker in bladder carcinoma: Correlation with tumor grade, stage and prognosis. Int Urol Nephrol 36: 401-405, 2004.

33. Deniz H, Karakök M, Yagci F and Güldür ME: Evaluation of relationship between HIF-1alpha immunoreactivity and stage, grade, angiogenic profile and proliferative index in bladder urothelial carcinomas. Int Urol Nephrol 42: 103-107, 2010.

34. Wakusawa R, Abe T, Sato H, Yoshida M, Kunikata H, Sato Y and Nishida K: Expression of vasohibin, an antiangiogenic factor, in human choroidal neovascular membranes. Am J Ophthalmol 146: $235-243,2008$ 